\title{
A STUDY OF VARIOUS DRUGS CAUSING FIXED DRUG ERUPTIONS
}

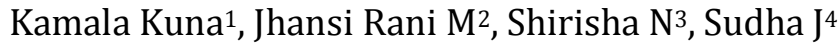

\section{HOW TO CITE THIS ARTICLE:}

Kamala Kuna, Jhansi Rani M, Shirisha N, Sudha J. "A Study of Various Drugs Causing Fixed Drug Eruptions". Journal of Evolution of Medical and Dental Sciences 2015; Vol. 4, Issue 52, June 29; Page: 8986-8992,

DOI: $10.14260 /$ jemds/2015/1304

ABSTRACT: AIM: To study the pattern of fixed drug eruptions to various drugs and to find out the common drugs causing fixed drug eruptions using provocative tests. 30 patients of FDE were studied. The pattern of fixed drug eruptions, most common class of drug, individual drugs causing positive reaction using provocation tests. Results were analyzed using suitable statistical methods. RESULTS: Drug eruption which is commonest in our study was fixed drug eruption (54.54\%). 66.66\% of cases had only cutaneous lesions. The commonest site of lesion was over limbs (46.66\%). Maximum number of patients showed positive reaction on provocative test with oxyphenbutazone followed by sulphamethoxazole. CONCLUSION: This study helps to investigate the drug causing FDR with particular clinical pattern more accurately, especially those with a multi-drug history. Knowledge of these drug eruptions, the causative drugs and the prognostic indicators is essential for clinicians for diagnosis and prevention of adverse drug reactions. Pharmacovigilance activity is significantly effective in increasing the reporting of ADRs.

KEYWORDS: adverse drug reactions, fixed drug eruptions, provocative tests.

INTRODUCTION: Adverse drug reactions are very common occur in 5\% of general population. A Cutaneous Adverse Drug Reaction (CADR) caused by a drug is an undesirable change in the structure or function of the skin, its appendages or mucous membranes. Cutaneous adverse drug reactions (CADR) are the commonest ADRs (30-45\%) and responsible for about $2 \%$ of hospital admissions.[1] Almost any medicine can induce skin reactions, but certain drug classes, such as non-steroidal antiinflammatory drugs (NSAIDs), antibiotics and antiepileptic drugs, have drug eruption rates approaching 1-5\%.[2] Brocq in 1984,[3] coined the term 'fixed eruption' to describe a pattern of skin eruption due to antipyrine. Fixed drug eruptions (FDE) were reported to be the most common cutaneous adverse drug reaction in an Indian report.[4]

Drugs implicated in causing FDE have changed over time and this study aims at understanding these trends. The Fixed drug eruptions (FDEs) are defined as recurrent lesions at the same (Skin or mucosal) sites after repeated intake of the causative agent.[5] FDEs usually present as itching or burning, well circumscribed, erythematous macules, patches, or plaques that leave hyperpigmentation after resolving. Vesicles or bullae may occasionally be seen. There are many causative agents. The incidence of FDE for a particular drug depends on the frequency of its use. Therefore, the list of etiologic drugs varies from one place to another and from time to time.[6] The list of causative drugs changes with time and prescribing patterns.

Techniques to identify the causative drug are intradermal testing, patch tests and invitro tests like flow cytometry and cytokine assays. But there are problems with the availability, applicability and reliability of these tests. It is now generally agreed that the only reliable method for finding the causative drugs is the provocation method.[7] The present study is an attempt to find out the drugs commonly causing fixed drug eruption in and around Visakhapatnam by provocation tests. 


\section{ORIGINAL ARTICLE}

OBJECTIVES: To study the pattern of Fixed Drug Eruptions due to various drugs among the patients attending the Department of Dermatology, King George Hospital, Visakhapatnam. To find out the drugs commonly causing Fixed Drug Eruption in those patients by provocation tests.

MATERIAL \& METHODS: The material for the present study comprises 30 cases of fixed drug eruption, who attended the Out Patient Department of Dermatology, King George Hospital, Visakhapatnam from 01-05-2007 to 31-04-2008. A Prospective observational study was done on selected patients.

A prospective study was undertaken in. Patients who were attending the Dermatology outpatient department with definite and clinically diagnosed drug eruptions were enrolled in the study. Consent of the patient was taken for the enrollment in the study. Patients with severe debilitating systemic diseases, pregnant women and who did not accept the informed consent form were excluded from the study. Study protocol was approved by institutional ethics committee. A detailed history of drug intake was taken. Accordingly, a list of suspected drugs was prepared for each patient. A complete clinical examination was done in all the patients and details recorded in the proforma enclosed. Patients from far off places were admitted in the ward for provocation tests. VDRL test was performed on all patients presenting with genital lesions and lesions at mucocutaneous junctions.

The patients were motivated to undergo provocation tests and warned of activation of the lesions following the administration of the offending drug. They were also reassured of the relative safety of provocation tests in fixed drug eruption. They were motivated to undergo provocation tests. The patients were warned of activation of the lesions following the administration of the offending drug. They were also reassured of the relative safety of provocation tests in fixed drug eruption. After preparation of the patient, drugs which are commonly known to cause fixed eruption in India were used in addition to the drugs suspected to have caused fixed eruption from the patient's history. Oxyphenbutazone, Phenylbutazone, Acetyl salicylic acid and Metimazole are some drugs used for provocative tests. Emergency drugs like adrenaline were kept to combat complications that might arise.

For testing sensitivity an adequate dose of the drug (Suspected from the patient's history to have caused the eruption) was given orally. Itching or burning along with erythema in the lesions, increase in the width compared to the width before provocation test and bulla formation is taken as criteria for a positive reaction either alone or in combination. Test for poly-sensitivity and cross sensitivity were also done. Poly sensitivity implies positive reaction with drugs belonging to different groups (Eg. If the patient had positive reaction with sulphamethoxazole, he was next tested with, tetracyclines). Cross sensitivity implies positive reaction to drugs belonging to the same group which originally caused the eruption. Eg: if the patient had a positive reaction with sulphamethoxazole, he was also tested with sulphadiazine, sulphadimethoxine and dapsone to detect any cross sensitivity.

RESULTS: A total of 55 cases of drug eruption were seen during the period of study out of which 30 cases were of FDE. These 30 cases were analyzed. Out of 30 patients, 20 cases $(66.66 \%)$ were males and 10cases (33.33\%) were females. Maximum patients belonged to the age group of 21-30 (Mean Age is 25.49 years).The most common pattern of cutaneous ADR observed was fixed drug eruption (54.54\%) followed by photosensitivity (5.45\%). (Fig. 1) 


\section{ORIGINAL ARTICLE}

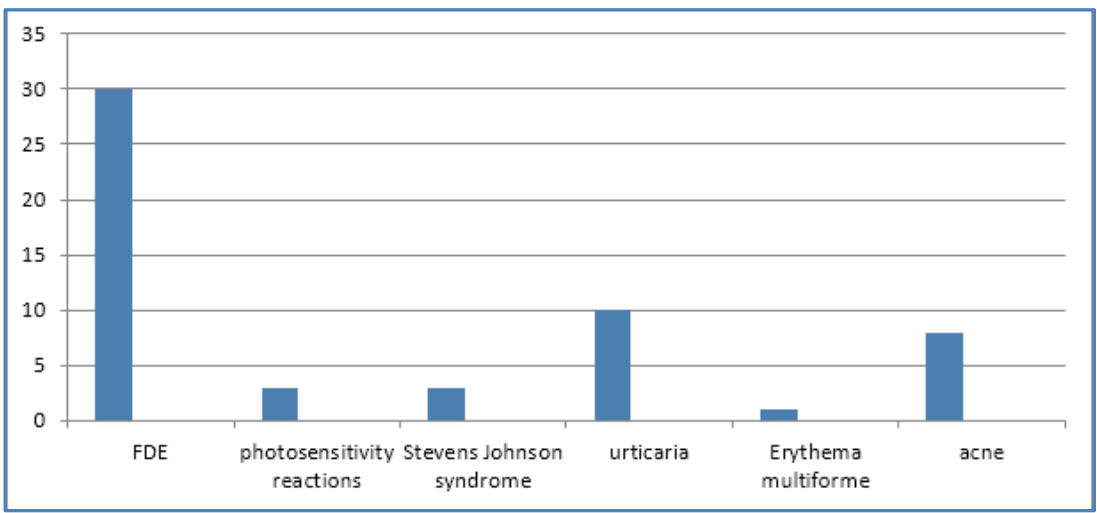

Fig. 1: Common pattern of cutaneous ADR

The presenting symptom in $40 \%$ of cases was itching, $33.33 \%$ of cases burning and itching, $6.66 \%$ of cases only burning sensation and $10 \%$ of cases were asymptomatic. (Fig: 2 )

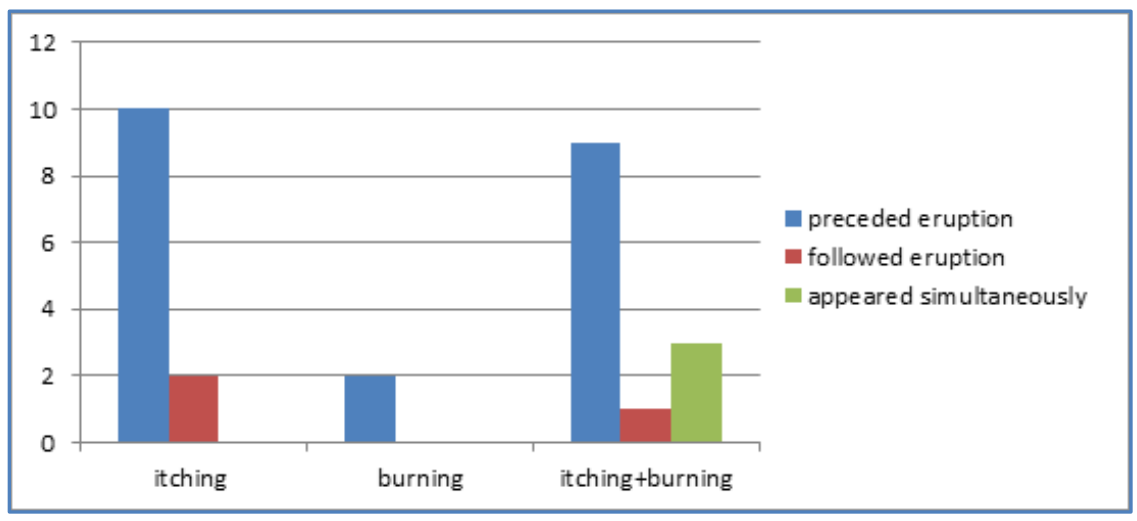

Fig. 2: Symptoms in FDE

$66.66 \%$ of cases had only cutaneous lesions, $13.33 \%$ of cases had no skin lesions. (Fig: 3 )

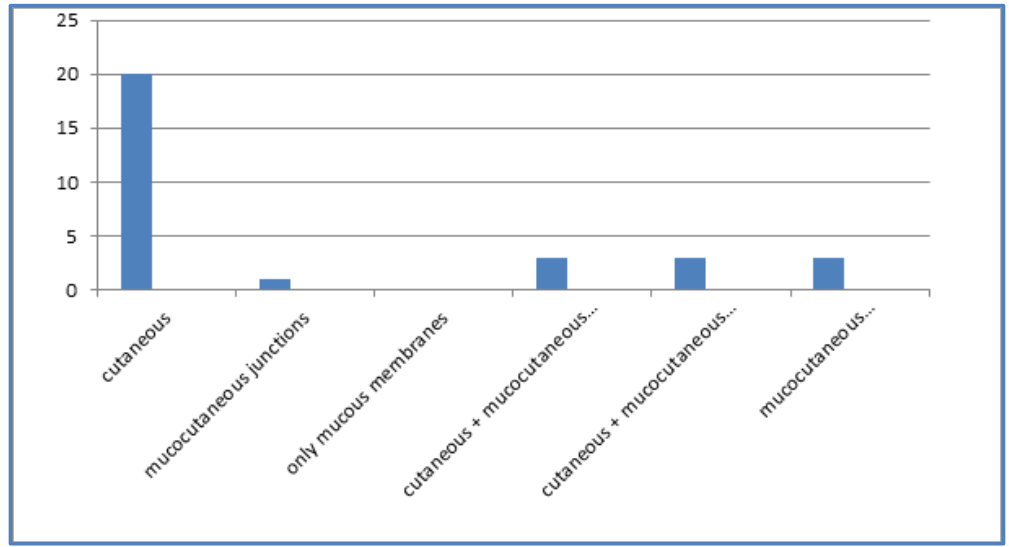

Fig. 3: Distribution of Lesions 


\section{ORIGINAL ARTICLE}

96.66\% of cases had pigmented macules, $16.66 \%$ of cases had a red zone around the pigmented macule, $16.66 \%$ of cases had bullae and $20 \%$ of cases had erosions.

Maximum number of patients (46.66\%) had lesions on the limbs followed by the trunk, mucocutaneous junction, head and neck.

The results of provocative tests showed positive reaction with oxyphenbutazone followed by sulphamethoxazole. Other drugs which showed positive reactions were phenylbutazone, analgin, sulphadiazine, and thiacetazone. (Tab: 1)

In one case the reaction following provocation tests was that no increase in the width of the zone of erythema. One case had a zone of erythema around the lesion $(0.1 \mathrm{~cm})$ when he first reported, he did not develop it after provocation test. In another case there is a zone of erythema of $0.3 \mathrm{cms}$ at the time of reporting. After the test the zone measured $0.1 \mathrm{cms}$. The rest of the patients did not have a zone of erythema before provocation test. Two cases had bullae at the time of examination. After provocation, they did not develop bullae.

Time interval that elapsed following drug intake and initiation of reaction in the lesions in majority of cases was between $1 / 2$ hour to 2 hours.

DISCUSSION: FDE is more common in men than in women in the ratio of 2: 1.Similar findings were encountered in Cheng-Han Lee etal study.[8] The commonest age group affected is 21-30 (36.66\%), followed by 11-20 years age group (23.33\%), i.e., FDE is commonly seen in adolescents and young adults. A similar higher incidence among 21 - 30 years age group was reported by J. Das (2001).[9]

Incidence of FDE among patients who attend the Department of Dermatology, King George Hospital, Visakhapatnam is $0.61 \%$. FDE is the commonest type of drug eruption seen in this hospital $(54.54 \%)$ followed by photosensitivity (5.45\%). Others have noted maculopapular rash. FDE is a common form of cutaneous adverse drug reactions whose exact etiology is unknown. pathogenesis: They are supposed to be caused by epidermal CD8 T cells, which are retained in the lesions forming an immunologic memory, which gets activated on re-challenge.[10] Intraepidermal CD8 Tcells with an effector-memory phenotype resident in Fixed drug eruption lesion have a major contributing role in pathogenesis. Majority of the patients (40\%) complained of itching as the major symptom of FDE and in a majority $(70 \%)$, the symptoms preceded the eruption. Local symptoms may include pruritus, burning, and pain.[11] Systemic symptoms are uncommon, but fever, malaise, nausea, diarrhea, abdominal cramps, anorexia, and dysuria have been reportedin other studies.[12]

Majority of the cases (66.66\%) had only cutaneous lesions.13.33\% of cases had no skin lesions. The most characteristic feature of FDE is reactivation of the inflammatory process in the previously involved site(s) with each subsequent exposure.[13] Red zone around pigmented macules is the common morphology of lesions in our study. The classic morphology of FDE lesion is dusky red painful patches that leaves long lasting or permanent deep post inflammatory hyperpigmentation.[13] Commonest sites affected in the present study are the limbs (86.66\%) followed by trunk. Lips are the most common site followed by genitalia for FDE in the study by Mahboob and Haroon.[13]

Provocation tests were done in all the cases with the suspected drugs. Results of provocative tests showed positive reaction with oxyphenbutazone $(33.15 \%)$ followed by sulphamethoxazole $(18.18 \%)$ in the present study. Drug classes, such as non-steroidal anti-inflammatory drugs (NSAIDs), antibiotics and antiepileptics, have drug eruption rates approaching 1-5\%.[14] Non-steroidal antiinflammatory drugs were the most common causative agents, accounting for $12.8 \%$ of cases, $10.3 \%$ caused by antibiotics in Cheng-Han Lee etal study. ${ }^{[8]}$ Cross sensitivity was found between 
oxyphenbutazone and phenylbutazone in 3 cases, but not among various sulphonamides. Gupta (2003) showed similar results.[3] Cross sensitivity tends to occur in other drugs similar in structure to the causative agent.[15]

FDE due to thiacetazone was observed in one patient.Mani et al.[16] Reported that thiacetazone was the most common antimicrobial drug to cause eruptions. The time latency between application and reaction ranged from $1 / 2$ hour to 10 hours with a mean of 2.36 hours. Time interval between reaction and provocation test should be at least 5 times the drug elimination in order to guarantee complete elimination. The reaction under investigation should have resolved completely, clinically and according to lab results-if measured initially and being abnormal. Any corrective medication or co-medication that might influence the outcome of the test result should be completely washed out. Whereas this happens within a few days with antihistamines or intravenous steroids for the treatment of systemic reactions, a sufficient wash out time for topical steroids for treatment of contact allergy might be up to 4 weeks.[17]

CONCLUSION: Our study highlighted the common occurrence of FDEs, and the range of causative drugs. Fixed drug eruption and acneform eruption are the most commonly encountered ACDRs at our tertiary care centre. Most common drugs causing fixed eruption are oxyphenbutazone followed by sulphamethoxazolecorticosteroids, isoniazid, rifampicin. Knowledge of these drug eruptions, the causative drugs and the prognostic indicators is essential for clinicians for diagnosis and prevention of adverse drug reactions. It is recommended to advise patients to carry a card or an emergency identification of offending drugs in their wallets that list the drug allergies and/or intolerances. Pharmacovigilance activity is significantly effective in increasing the reporting of ADRs.

ACKNOWLEDGEMENT: Authors acknowledge the great help received from the scholars whose articles cited and included in references of this manuscript. The authors are also grateful to authors / editors/publishers of all those articles, journals and books from where the literature for this article has been reviewed and discussed.

\section{REFERENCES:}

1. Valeyrie-Allanore L, Sassolas B, Roujeau JC. Drug-induced skin, nail and hair disorders. Drug Saf. 2007; 30: 1011-1030.

2. Bigby M. Rates of cutaneous reactions to drugs. Arch Dermatol 2001; 137: 765-770.

3. Gupta R. Drug causing fixed drug eruption: confirmed by provocation tests. Indian J Dermatol Venereol Leprol 2003; 69: 120-121.

4. Pudukadan D, Thappa DM. Adverse cutaneous drug reactions: Clinical pattern and causative agents in a tertiary care center in South India. Indian J Dermatol Venereol Leprol 2004; 70: 20-4.

5. Ozkaya E. Fixed drug eruption: state of the art. J Dtsch Dermatol Ges 2008; 6: $181 \mathrm{e} 8$.

6. Sehgal VN, Srivastava G. Fixed drug eruption (FDE): changing scenario of incriminating drugs. Int J Dermatol 2006; 45: 897e908.

7. Ramam M, Kumar U, Bhat R, Sharma V. K. Oral drug provocation test to generate a list of safe drugs: Experience with 100 patients. Indian J Dermatology Venereology Leprosy 2012; 78: 595-8. 


\section{ORIGINAL ARTICLE}

8. Cheng-Han Lee 1, Yi-Chun Chen 2, Yung-Tsu Cho 1, Chia-Ying Chang 1, Chia-Yu Chu 1, * Fixeddrug eruption: A retrospective study in a single referral center in northern Taiwan. DERMATOLOGICA SINICA 30 (2012) 11e15.

9. Das J, Mandal A C. A study of drug eruptions by provocative tests. Indian J Dermatol Venereol Leprol 2001; 67: 238-9.Rahmeh Fayez (2011) Drugs Causing Fixed Drug Eruption: A Clinical Study Rahmeh Fayez MD*, Nidal Obaidat MD*, Ayman Al-Qa'qaa' MD*, Basil Al-Rawashdeh MD*, Manal Ma'aita BSc**, Nada Al-Azab BSc** JRMS September 2011, 18(3): 16-20.

10. Kauppinen K, Stubb S. Fixed eruptions: Causative drugs and challenge tests. Br J Dermatol 1985; 112: 575-8.

11. Freedberg IM, Eisen AZ, Wolff K, Austen KF, Goldsmith LA, Katz SI. Fixed drug eruptions. In: Fitzpatrick's Dermatology in General Medicine. $6^{\text {th }}$ ed. New York, NY: McGraw-Hill; 2003: 1333.

12. Sehgal V. N, Srivastava G. Fixed drug eruption (FDE): changing scenario of incriminating drugs. Int J Dermatol. Aug 2006; 45(8): 897-908.

13. Mahboob A, Haroon TS. Drugs causing fixed drug eruption: a study of 450 cases. Int J Dermatol 1998; 37: 833-838.

14. Bigby M. Rates of cutaneous reactions to drugs. Arch Dermatol 2001; 137: 765-770.

15. Sanmukhani J, Shah V, Baxi S, Tripathi C. Fixed drug eruption with ornidazole having crosssensitivity to secnidazole but not to other nitro-imidazole compounds: A case report. Br J Clin Pharmacol 2010; 69: 703-44.

16. Mani M. Z, Mary Mathew. A study of 218 drug eruptions. Indian J DermatolVenereolLepr1983; 49: 109 - 117.

17. Barbaud A, Goncalo M, Bruynzeel D, Bircher A. Guidelines for performing skin tests with drugs in the investigation of cutaneous adverse drug reactions. Contact Dermatitis 2001; 45: 321328.

\begin{tabular}{|c|c|c|}
\hline Drugs & No. Tested & No. Positive \\
\hline Oxyphenbutazone & 16 & 07 \\
\hline Phenylbutazone & 08 & 03 \\
\hline Acetyl salicylic acid & 09 & --- \\
\hline Analgin & 13 & 01 \\
\hline Sulphadiazine & 09 & 01 \\
\hline Sulphadimethoxine & 07 & --- \\
\hline Trimethoprim & 11 & --- \\
\hline Sulphamethoxazole & 11 & 02 \\
\hline Dapsone & 08 & --- \\
\hline Tetracycline & 09 & --- \\
\hline Oxytetracycline & 06 & --- \\
\hline Doxycycline & 08 & --- \\
\hline Paracetamol & 02 & --- \\
\hline Isonicotinic acid hydrazide & 01 & --- \\
\hline Thiacetazone & 01 & 01 \\
\hline Procaine penicillin & 08 & --- \\
\hline Table 1 & \\
\hline
\end{tabular}




\section{ORIGINAL ARTICLE}

\section{FIXED DRUG ERUPTION:}

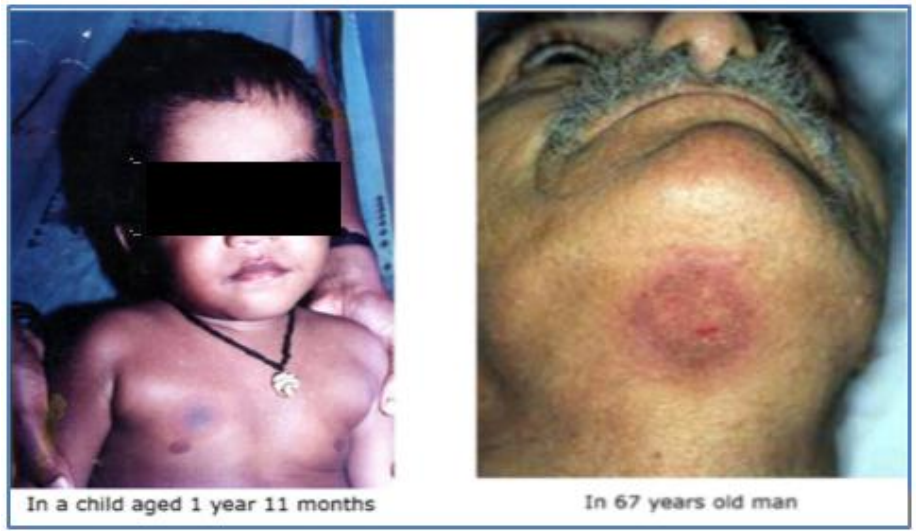

\section{ZONE OF ERYTHEMA AROUND THE LESIONS SEEN AFTER PROVOCATION TEST:}

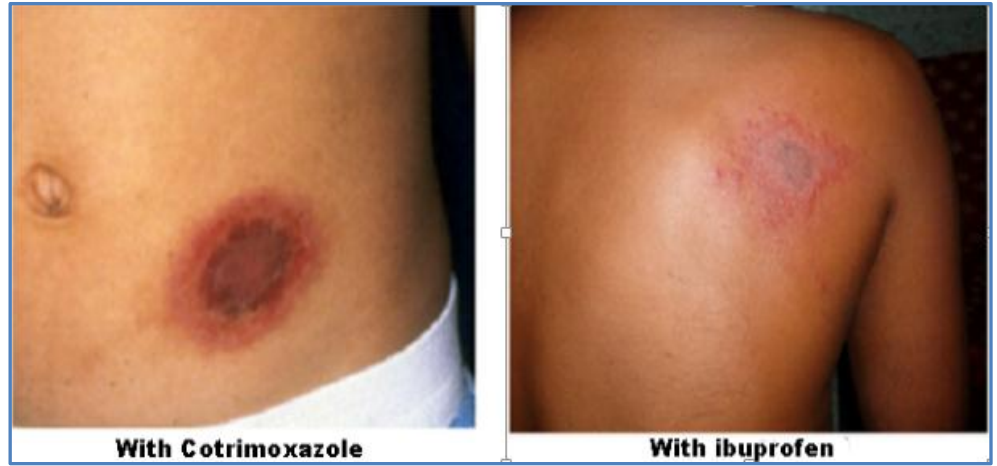

\section{AUTHORS:}

1. Kamala Kuna

2. Jhansi Rani M.

3. Shirisha N.

4. Sudha J.

\section{PARTICULARS OF CONTRIBUTORS:}

1. Assistant Professor, Department of Pharmacology, Andhra Medical College, Visakhapatnam.

2. Assistant Professor, Department of Pharmacology, Andhra Medical College, Visakhapatnam.

3. Senior Resident, Department of Pharmacology, Andhra Medical College, Visakhapatnam.

\section{FINANCIAL OR OTHER} COMPETING INTERESTS: None
4. Professor, Department of Pharmacology, Andhra Medical College, Visakhapatnam.

\section{NAME ADDRESS EMAIL ID OF THE CORRESPONDING AUTHOR:}

Dr. Kamala Kuna,

Door No. 39-1-27/2,

Murali Nagar, Visakhapatnam-530007,

Andhra Pradesh, India.

E-mail: kamalakuna@gmail.com

Date of Submission: 05/06/2015.

Date of Peer Review: 08/06/2015.

Date of Acceptance: 20/06/2015.

Date of Publishing: 26/06/2015. 